\title{
可溶液加工的蒽醌/芴类双极性荧光材料的合成及其光电性质
}

陈 吴 ${ }^{1}$ 杨 涛 $^{1}$ 李杰伟 ${ }^{2}$ 张新稳 ${ }^{1}$ 钱 妍 ${ }^{1, *}$ 解令海 ${ }^{1}$ 黄 维 ${ }^{1,2, *}$

( ${ }^{1}$ 南京邮电大学信息材料与纳米技术研究院暨有机电子与信息显示国家重点实验室培育基地,

先进生物与化学制造协同创新分中心, 南京 210023; “南京工业大学先进材料研究院暨柔性电子重点实验室,

先进生物与化学制造协同创新中心，南京 211816)

\begin{abstract}
摘要: 通过 Suzuki 反应合成得到了一种可溶液加工的葸醌/茀类双极性荧光材料 2-葸醌基-9,9'-二异辛基苆 (FAA), 利用紫外吸收光谱和荧光发射光谱对其光物理性质进行了初步研究, 并采用密度泛函理论计算方法 分析了分子光物理性质的本质。通过单载流子器件的性能测试, 证实了 FAA 具有较好的双极性传输特性。进 而研究了该材料的电致发光性能, 将其掺杂于主体材料 1,3-双 (9-咔唑基)苯(mCP)中, 利用旋涂法制备了结 构为ITO(氧化铟锡)/PEDOT:PSS (聚 3,4-乙撑二氧噻吩: 聚苯乙烯磺酸盐)/mCP:FAA/TmPyPb (1,3,5-三 [(3-吡 啶基)-3-苯基]苯)/LiF/AI 的有机发光二极管。器件的启亮电压约为 $7.4 \mathrm{~V}$, 最大亮度为 $1719 \mathrm{~cd} \cdot \mathrm{m}^{-2}$, 最大电流 效率和最大功率效率分别为 $1.66 \mathrm{~cd} \cdot \mathrm{A}^{-1}$ 和 $0.56 \mathrm{Im} \cdot \mathrm{W}^{-1}$; 同时, 结合器件各功能层的能级结构图, 探讨了其 电致发光机制。
\end{abstract}

关键词: 有机发光二极管; 可溶液加工; 芴类荧光材料; 双极性特性; 葱醌 中图分类号: 0649

\section{Synthesis and Optoelectronic Properties of a Solution- Processable Anthraquinone/Fluorene Hybrid Bipolar Fluorescent Material}

\author{
CHEN Hao ${ }^{1} \quad$ YANG Tao $^{1} \quad$ LI Jie-Wei ${ }^{2} \quad$ ZHANG Xin-Wen ${ }^{1} \quad$ QIAN Yan ${ }^{1, *}$ \\ XIE Ling-Hai ${ }^{1} \quad$ HUANG Wei ${ }^{1,2, *}$
}

('Key Laboratory for Organic Electronics and Information Displays \& Institute of Advanced Materials, Jiangsu National Synergetic Innovation Center for Advanced Materials, Nanjing University of Posts \& Telecommunications, Nanjing 210023, P. R. China; ${ }^{2}$ Key Laboratory of Flexible Electronics \& Institute of Advanced Materials, Jiangsu National Synergetic Innovation Center for Advanced Materials, Nanjing Tech University, Nanjing 211816, P. R. China)

\begin{abstract}
The solution-processable, anthraquinone-based, fluorene bipolar fluorescent material 2-(9,9'-bis (2-ethylhexyl)-9H-fluoren-2-yl)anthracene-9,10-dione (FAA) was synthesized via a Suzuki reaction. The photophysical properties of FAA were subsequently investigated by acquiring absorption and photoluminescence spectra, and its optical properties were studied using computational density functional theory methods. Data obtained from single-carrier devices incorporating FAA demonstrated its well-matched bipolar charge-transport characteristics. The electroluminescence performance of this material was also examined by doping FAA into a 1,3-di(9H-carbazol-9-yl)benzene (mCP) matrix as the light-emitting layer via spin coating to produce an organic
\end{abstract}

Received: March 21, 2016; Revised: May 16, 2016; Published online: May 16, 2016.

*Corresponding authors. QIAN Yan, Email: iamyqian@njupt.edu.cn. HUANG Wei, Email: iamwhuang@njupt.edu.cn.

The project was supported by the National Key Basic Research Program of China (973) (2012CB723402), Jiangsu National Synergistic Innovation Center (2011) for Advanced Materials (SICAM), China, Priority Academic Program Development of Jiangsu Higher Education Institutions (PAPD), China (YX03001), Synergistic Innovation Center for Organic Electronics and Information Displays, China, National Natural Science Foundation of China (21373114, 21573111), and Qing Lan Project and NUPT 1311 Project, China.

国家重点基础研究发展规划项目(973) (2012CB723402), 先进生物与化学制造协同创新中心(国家级 2011 协同创新中心), 江苏高校优势学科 建设工程(YX03001), 江苏省有机电子和信息显示协同创新中心, 国家自然科学基金(21373114, 21573111)及江苏省青蓝工程与南京邮电大学 “ $1311 ”$ 人才项目资助 
light-emitting diode (OLED) with an indium tin oxide (ITO)/poly(3,4-ethylenedioxythiophene:poly(styrenesulfonate) (PEDOT:PSS)/mCP:FAA/3,3'-(5'-(3-(pyridin-3-yl)phenyl)-[1,1':3',1"-terphen-yl]-3,3"-diyl)dipyridine (TmPyPb)/ $\mathrm{LiF} / \mathrm{Al}$ structure. This device exhibited a maximum luminance of $1719 \mathrm{~cd} \cdot \mathrm{m}^{-2}$ with a turn-on voltage of $7.4 \mathrm{~V}$, along with maximum current and power efficiencies of $1.66 \mathrm{~cd} \cdot \mathrm{A}^{-1}$ and $0.56 \mathrm{Im} \cdot \mathrm{W}^{-1}$, respectively. The electroluminescence mechanism of the OLED is discussed based on the energy level diagrams of the functional layers.

Key Words: Organic light-emitting diode; Solution processing; Fluorescent material; Bipolar characteristics; Anthraquinone

\section{1 引 言}

有机发光二极管(OLED)由于具有全色彩自发 光、高对比度、宽视角、低驱动电压、低功耗、 快速响应性、可柔性制备等优势，使其在平板显 示和固态照明领域中受到了广泛关注 ${ }^{1-5}$ 。迄今为 止，大多数高效率的小分子 OLED仍然是通过真空 蒸发沉积多层器件结构制备 ${ }^{6-9}$, 这种方法所需的 设备和工艺比较复杂，对有机材料的利用率也很 低，从而导致 OLED产业化制造成本过高 ${ }^{6,9,10}$ 。同 时，像素点加工时蒸镀掩模的使用，在很大程度 上限制了 OLED 的尺寸扩展性和高分辨率应 用 $6,11,12$ 。相比之下，采用溶液加工法例如旋涂、挤 压式涂布、喷墨打印和丝网印刷等 ${ }^{6,10-14}$ ，可实现低 成本和大面积的柔性 OLED制造。溶液法使用的设 备简单、费用低, 且材料利用率高, 能够大大降 低 OLED生产成本 ${ }^{11,13,15}$ 。而且, 溶液法具有易于扩 展的特性, 也无需精细金属掩模, 使得大面积 OLED 制备成为可能 ${ }^{13,16}$ 。此外, 溶液法能够有效 地控制掺杂材料的比例和浓度，还可实现单一发 光层中多个客体与主体的共掺杂 OLED 器件 ${ }^{6,15}$ 。

有机功能材料对于 OLED 的器件性能好坏起着 至关重要的作用, 国内外众多科研工作者和企业 为此长期不断努力研究和开发综合性能优异的新 型材料。其中, 双极性材料由于能同时传输电子 和空穴两种载流子, 通常被用于有机发光层中调 控 OLED 的载流子平衡, 拓宽载流子的复合区域, 保证良好的器件性能和操作稳定性 ${ }^{11,17-25}$ 。此外, 双极性材料还可能简化器件结构, 获得双层甚至 单层的理想器件, 降低制造成本 ${ }^{17,19-21,24-26}$ 。如今, 越来越多的可溶液加工小分子双极性主体材料已 经被投入到溶液法制备的主客体掺杂 OLED中，提 高该类器件的发光效率和稳定性, 并取得了巨大 的进展 7,9-12,22,26,27。但是, 关于小分子双极性苂光材 料作为发光体应用于溶液法制备 OLED 的报道却为 数不多 ${ }^{28-31}$ 。性能优异的小分子双极性菼光材料不
仅能够有效改善 OLED 中载流子的平衡, 简化器件 结构 ${ }^{17,25,32}$; 还可直接作为发光材料在非掺杂的情况 下得到高效率的 OLED 器件 17,18,25,29,32-37, 避免了掺杂 体系容易产生相分离的问题。因此, 发展可溶液 加工的小分子双极性苂光材料用以制备简单、高 效率的溶液法加工 OLED 具有重要的科学以及应用 意义。

在同一个分子中引入给电子和吸电子基团分 别作为空穴和电子传输单元, 构成 D-A (donor-acceptor)型的分子结构是获得高性能的双极性苂光材 料行之有效的方法 ${ }^{18,25,29,32,33,36}$ 。D-A 型双极性苂光材 料在激发态时由于分子内电荷转移(intramolecular charge transfer, ICT)作用会产生有效的辐射发光， 因而使其能够拥有很高的荧光量子效率 32,38 。在众 多的有机电致发光材料中，芴类化合物由于具有 刚性结构, 苂光量子效率高和可修饰性强等优点 而倍受关注 ${ }^{39-44}$ 。但是，该类化合物的电子亲和势 比较低, 使得电子不易注入, 其电子传输性能也 远低于空穴传输性能。这种载流子注入与传输的 不平衡, 将直接导致 OLED 器件发光效率的降低。 为了解决上述问题，人们通常将合适的强吸电子 基团引入芴类化合物中进行修饰，使其成为 D-A 型双极性苂光材料，从而降低该类化合物的电子 注入势垒, 以提高电子注入能力, 同时增强其电 子传输性能, 实现载流子注入与传输的平衡, 进 而提升 OLED 器件性能 $34,45-49$ 。

最近, Adachi 等 ${ }^{50}$ 合成了一系列具有 D-A-D型 结构的小分子葱醌类衍生物, 并将它们掺杂到主 体材料中作为有机发光层, 通过真空蒸镀制备得 到了高效率延迟荧光 OLED 器件，其性能可与磷光 器件媲美。其中, 蒽醌基团对于调控上述材料中 载流子的注入与传输平衡起到了关键作用。基于 此, 我们尝试向芴中引入葱醌基团, 改善其电子 注入和传输性能，同时在芴的 9 位上用烷基链取 代, 增加材料的溶解性, 构筑了一种可溶液加工 
的具有 D-A 型结构以及双极传输特性的蒽醌芴类 材料 2-葸醌基-9,9'-二异辛基芴(FAA), 并初步探索 了该材料的双极性传输以及电致发光性能。

\section{2 实验部分}

\section{1 仪器和试剂}

Bruker AV400 核磁共振波谱仪(德国布鲁克公 司); Bruker autoflex speed MALDI-TOF 质谱仪(德 国布鲁克公司); Shimadzu UV-3600 紫外-可见分光 光度计 (日本岛津公司); PerkinElmer LS 55 苂光分 光光度计(美国珀金埃尔默公司); Carlo Erba-1106 元素分析仪 (意大利卡劳尔巴公司); Bruker DektakXT 台阶仪(德国布鲁克公司); ZHD-300F 蒸 镀机; TDF-5227B复合真空计; 上海泰尧真空科技 有限公司膜厚监测仪; ST-86LA 屏幕亮度计; PR655 亮度光谱仪。

2-澳葱醌购自吉林奥来德光电材料股份有限公 司; 四(三苯基膦)钯购自百灵威科技有限公司; 氧 化铟锡(ITO)玻璃基板购自深圳南玻集团; PEDOT: PSS (型号为 AI4083) 购自德国拜耳公司; $\mathrm{mCP}$ 、 $\mathrm{TmPyPb} 、 N, N^{\prime}$-二苯基- $N, N^{\prime}$-二(1-萗基)- $1,1^{\prime}$ - 联苯4,4'-二胺(NPB)、1,3,5-三(1-苯基- $1 H$-苯并咪唑-2基)苯(TPBi) 和 $\mathrm{LiF}$ 均购自台湾 Nichem 公司; 其它 试剂均为市售分析纯试剂。

\subsection{2-葸醌基-9,9'-二异辛基芴的合成}

2-葱醌基-9,9'-二异辛基芴(FAA)的合成路线如 图 1 所示。其中，化合物 2-[2-(4,4,5,5-四甲基[1,3,2] 二氧杂环戊硼基)]-9,9'-二异辛基芴(1)的合成方法 可参考相关文献资料 ${ }^{51,52}$ 。在 $50 \mathrm{~mL}$ 两口反应瓶 中, 加入 2-溴葱醌( $0.36 \mathrm{~g}, 1.25 \mathrm{mmol})$ 和 2-[2-(4,4, 5,5-四甲基[1,3,2]二氧杂环戊嗍基)]-9,9'-二异辛基 芴 $(0.77 \mathrm{~g}, 1.5 \mathrm{mmol})$, 抽真空充氮气三次; 然后 快速加入 $50 \mathrm{mg}$ 四(三苯基膦)钯至反应瓶中。用注
射器分别抽取 $15 \mathrm{~mL}$ 甲苯/四氢呋喃混合溶剂(1:1) 和 $3 \mathrm{~mL}$ 碳酸钾/氟化钾混合溶剂 $\left(2 \mathrm{~mol} \cdot \mathrm{L}^{-1}\right)$ 打入反 应瓶中, 加热至 $90^{\circ} \mathrm{C}$, 摚拌反应 $48 \mathrm{~h}$ 。反应完成 后, 加水猝灭反应, 用二氯甲烷萃取反应液, 取 下层有机相; 旋蒸除去溶剂后, 经硅胶柱层析提纯 得到 $0.61 \mathrm{~g}$ 黄色粘稠物, 产率为 $81.3 \%$ 。 ${ }^{1} \mathrm{H}$ NMR $\left(400 \mathrm{MHz}, \mathrm{CDCl}_{3}\right): \delta 8.60(\mathrm{~s}, 1 \mathrm{H}), \quad 8.42-8.33$ $(\mathrm{m}, 3 \mathrm{H}), 8.09-8.04(\mathrm{~m}, 1 \mathrm{H}), 7.85-7.63(\mathrm{~m}$, $6 \mathrm{H}), \quad 7.43-7.30(\mathrm{~m}, 2 \mathrm{H}), 7.21-7.14(\mathrm{~m}, 1 \mathrm{H})$, $2.13-1.98(\mathrm{~m}, 4 \mathrm{H}), 0.98-0.64(\mathrm{~m}, 19 \mathrm{H}), 0.62-$ $0.48(\mathrm{~m}, 11 \mathrm{H}) ;{ }^{13} \mathrm{C}$ NMR $\left(100 \mathrm{MHz}, \mathrm{CDCl}_{3}\right)$ : $\delta$ 183.4, 183.0, 151.6, 150.8, 147.5, 142.6, $142.3, \quad 142.1, \quad 140.5, \quad 138.1, \quad 137.0, \quad 136.4$, $134.1, \quad 133.7, \quad 132.3, \quad 131.9, \quad 128.1, \quad 127.2$, $127.0, \quad 126.3, \quad 125.3, \quad 124.2, \quad 122.8, \quad 120.3$, 120.0, 119.9, 55.1, 44.4, 34.7, 33.8, 28.1, $27.1,22.7,14.0,10.4$; MALDI-TOF $m / z: 597.69$ $[\mathrm{M}+\mathrm{H}]^{+} ; \mathrm{C}_{43} \mathrm{H}_{48} \mathrm{O}_{2}(596.37)$ 元素分析计算值 $(\%)$ : C, $86.53 ; \mathrm{H}, 8.11$; 实测值 $(\%)$ : $\mathrm{C}, 86.76 ; \mathrm{H}$, 7.88 。

\section{3 理论计算方法}

FAA 分子的基态几何优化采用 M06-2X 方法在 6-311G $(d, p)$ 水平下进行。基于优化的几何结构采 用 TD-M06-2X方法在相同的水平下进行紫外-可见 吸收光谱的计算。激发态的优化采用 TD-B3LYP方 法在 6-31G $(d)$ 水平下进行。荧光光谱的计算采用 B3LYP 方法在 6-311G $(d, p)$ 水平下进行。电子跃迁 行为使用自然键跃迁轨道(NTO)进行分析。以上所 有的计算均采用高精度的量子化学程序 Gaussian $09^{53}$ 完成。

\section{4 器件的制备}

将刻蚀好的 ITO 玻璃基片(附着 $10 \Omega$ 方块电阻) 依次用专用清洗液、丙酮/乙醇混合液和去离子水

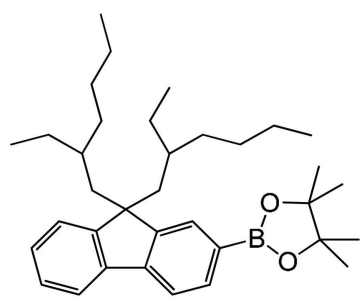

1

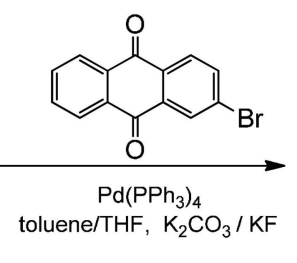

图 1 FAA 的合成路线

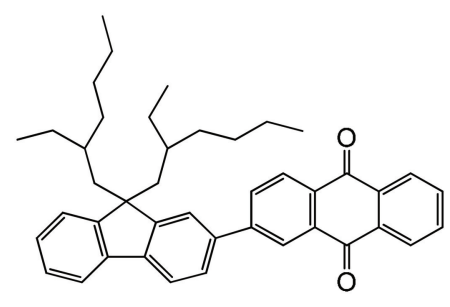

FAA

Fig.1 Synthetic route of FAA

FAA: 2-(9,9-bis(2-ethylhexyl)-9H-fluoren-2-yl)anthracene-9,10-dione; THF: tetrahydrofuran 
超声清洗后, 置于 $120{ }^{\circ} \mathrm{C}$ 的恒温干燥箱中干燥 1 $\mathrm{h}$, 再对其表面进行等离子体处理 $5 \mathrm{~min}$ 。采用溶液 和真空蒸镀相结合的方法制备 OLED 器件，以 ITO 为祄底, 发光层通过旋转涂膜法制备(膜厚由 Bruker DektakXT 台阶仪测定)，其它的有机层和阴极都 在 $5 \times 10^{-4} \mathrm{~Pa}$ 真空腔中采用常规的热阻加热方法蒸 发至祄底(膜厚由石英晶体振荡器监控)。PEDOT: $\mathrm{PSS} 、 \mathrm{mCP} 、 \mathrm{TmPyPb}$ 分别作为空穴注入层、主体 材料和电子传输层, $\mathrm{LiF} / \mathrm{Al}$ 为复合电极。

实验制备的器件结构为 ITO/PEDOT:PSS (40 $\mathrm{nm}) / \mathrm{mCP}: \mathrm{FAA}(8 \%(w)) / \mathrm{TmPyPb}(60 \mathrm{~nm}) / \mathrm{LiF}(0.8$ $\mathrm{nm}) / \mathrm{Al}(100 \mathrm{~nm})$, 其中 $w$ 为有机发光层掺杂 FAA 的 质量分数; 实验所用有机材料分子的化学结构式 和器件的结构如图 2 所示。器件的测试是在室温大 气环境下进行, 其发光面积是 $3 \mathrm{~mm} \times 4 \mathrm{~mm}$, 使用 Keithley 2602 型直流电源提供驱动电压并作为电流 源表, PR-650 亮度光谱仪测量电致发光光谱及亮 度参数, 光学平台等辅助仪器测量相关电致发光 性质, 从而得到了器件的电流密度一电压一亮度特 性曲线、电致发光光谱图、 $\operatorname{CIE}$ (国际照明委员会) 色坐标和电流效率-电流密度-功率效率特性曲线 等光电特性。

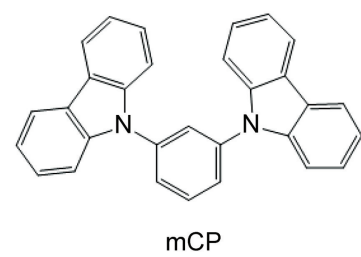

\begin{tabular}{|c|}
\hline LiF/AI \\
\hline TmPyPb \\
\hline mCP:FAA \\
\hline PEDOT:PSS \\
\hline ITO \\
\hline
\end{tabular}
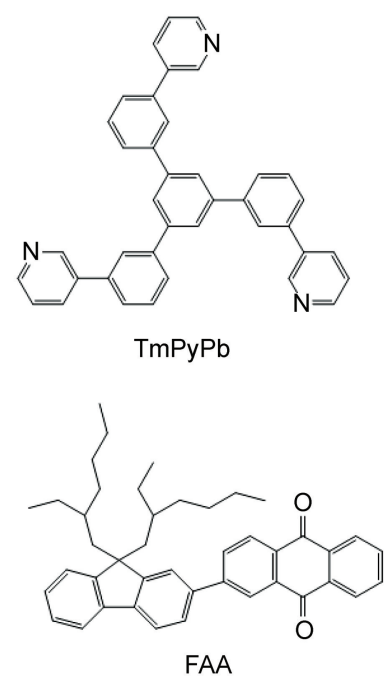

图 2 实验中所用有机材料分子的化学结构式和 器件的结构图

Fig.2 Chemical structures of organic materials and simplified configuration of OLED device

TmPyPb: 3,3'-(5'-(3-(pyridin-3-yl)phenyl)-[1,1':3',1'-terphenyl]-3,3"diyl)dipyridine; mCP: 1,3-di(9H-carbazol-9-yl)benzene;

PEDOT:PSS: poly(3,4-ethylenedioxythiophene:poly(styrenesulfonate); ITO: indium tin oxide; OLED: organic light-emitting diode

\section{3 结果与讨论}

\section{1 光物理性质}

图 3 是 FAA 在四氢呋喃溶液中和固态薄膜时的 归一化紫外可见吸收光谱和荧光发射光谱。FAA 溶液和薄膜吸收光谱的形状与峰位置都很接近, 都在 285-355 nm 和 365-465 nm 处有两个主要的 吸收带, 二者的最大吸收波长分别为 307 和 308 $\mathrm{nm}$ 。分别以 307 和 $308 \mathrm{~nm}$ 作为激发波长, 测试得 到了FAA 溶液和薄膜的苂光发射光谱; FAA 的溶 液和薄膜均具有单一黄绿光发射峰, 峰值分别为 540 和 $526 \mathrm{~nm}$, 半峰宽都在 $80 \mathrm{~nm}$ 左右。与薄膜相 比, FAA 溶液的荧光发射峰红移了 $14 \mathrm{~nm}$, 这是由 于FAA 分子在溶剂中的溶剂化效应很强, 减弱了 其受激分子荧光发射的能量，从而使得发射光波 长红移。为进一步理解 FAA 光物理性质的本质, 本文采用密度泛函理论计算了单分子的吸收和发 射光谱, 如图 4 所示。FAA 在 $395 \mathrm{~nm}$ 处的吸收峰 (理论计算为 $380 \mathrm{~nm}$ ) 可以归属为蒽醌占据轨道到非 占据轨道的局域 $n-\pi^{*}$ 跃迁。而在 $540 \mathrm{~nm}$ 处(理论 计算为 $558 \mathrm{~nm}$ )的发射峰可以归属为葱醌基团到芴 以及芴与葱醌共轭部分的部分扭曲分子内电荷转 移(quasi-TICT)跃迁, 这主要是由于激发态几何结 构相对于基态结构具有一定变化, 葱醌和芴基团 之间的夹角变小产生一定共轭的原因。

主客体掺杂器件中的能量传递, 要求主体材 料的发射光谱与客体材料的紫外可见吸收光谱之 间有较大程度的重叠，从而保证主客体间有效的 能量传递。从图 5 可以看出, 主体材料 $\mathrm{mCP}$ 薄膜 的发射光谱与客体材料 FAA 薄膜的吸收光谱具有 很好的光谱重叠。其中, $\mathrm{mCP}$ 薄膜的发射带很 宽, 在 369 和 $395 \mathrm{~nm}$ 处有两个发射峰(激发波长为

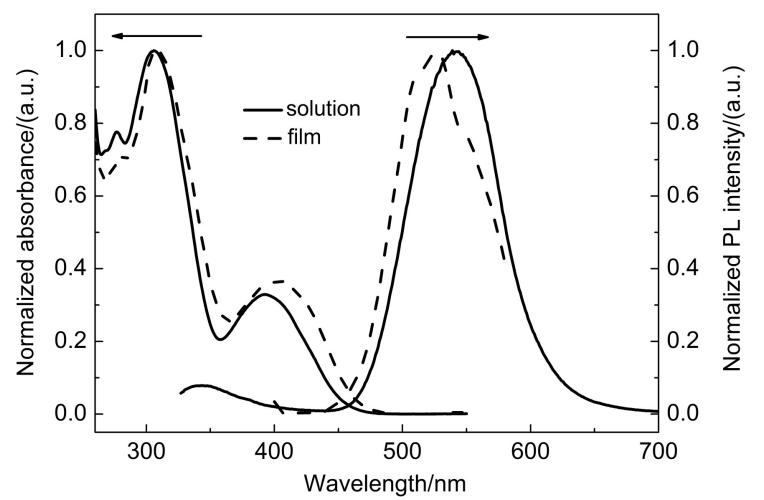

图 3 FAA 溶液(THF)和薄膜的归一化吸收和荧光发射光谱

Fig.3 Normalized absorption and fluorescence emission spectra of FAA in solution (THF) and film 


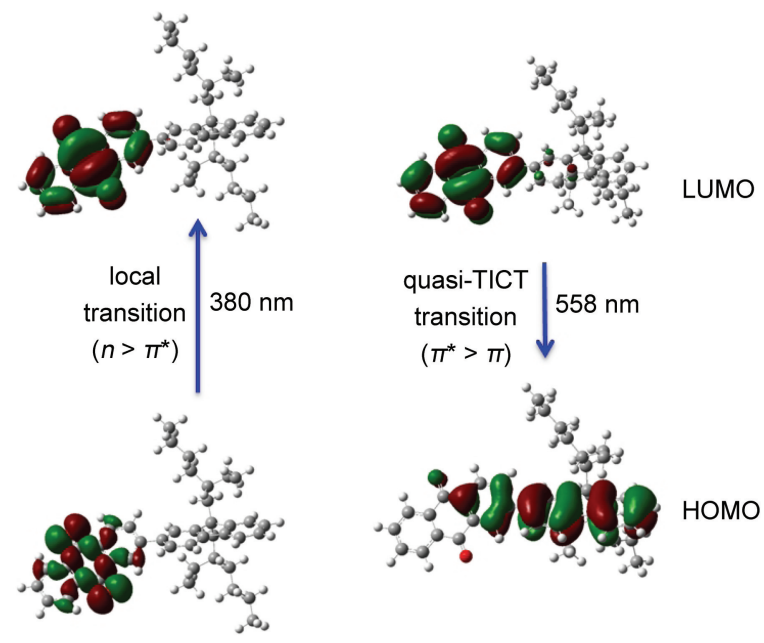

图 4 FAA 吸收与发射电子跃迁行为的自然键跃迁轨道分析

Fig.4 Electron transition of FFA for absorption and emission analyzed by natural transition orbitals (NTO) method

HOMO: the highest occupied molecular orbital; LUMO: the lowest unoccupied molecular orbital; TICT: twisted intramolecular charge transfer

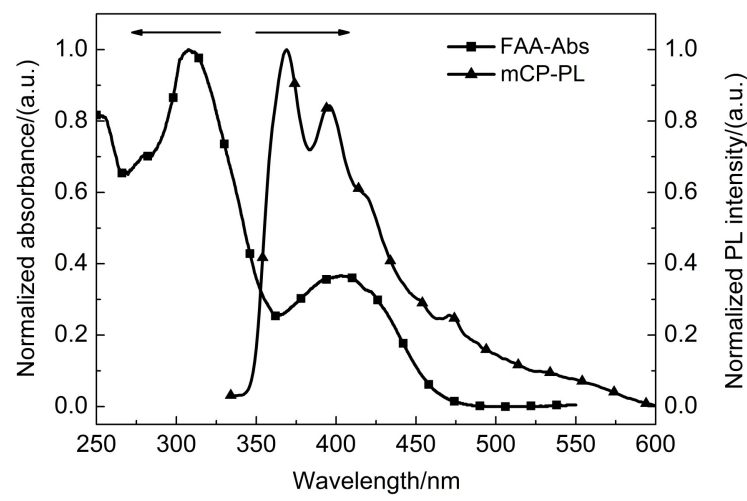

图 5 FAA 薄膜的归一化吸收光谱和 $\mathrm{mCP}$ 薄膜的 归一化发射光谱

Fig.5 Normalized absorption spectrum of FAA film and normalized fluorescence spectrum of $\mathrm{mCP}$ film Abs: absorbance; PL: photoluminescence

$314 \mathrm{~nm})$, 这与 FAA 薄膜在 $403 \mathrm{~nm}$ 处的吸收峰交叠 得较为完全, 说明主客体之间可以进行有效的能 量传递。

\section{2 电化学性质}

为了估算FAA 最高占据分子轨道 $(\mathrm{HOMO})$ 和最 低未占据分子轨道(LUMO)的能级, 本文利用循环 伏安法 $(\mathrm{CV})$ 对化合物进行了电化学分析。测试以 二茂铁为内标, 以无水四氢呋喃和二氯甲烷溶剂 分别测试还原和氧化电势, 以 $0.1 \mathrm{~mol} \cdot \mathrm{L}^{-1}$ 六氟磷 酸四丁基铵 $\left(\mathrm{Bu}_{4} \mathrm{NPF}_{6}\right)$ 为电解质。图 6 为 $\mathrm{FAA}$ 的循 环伏安曲线, 根据曲线可知该化合物的氧化电势

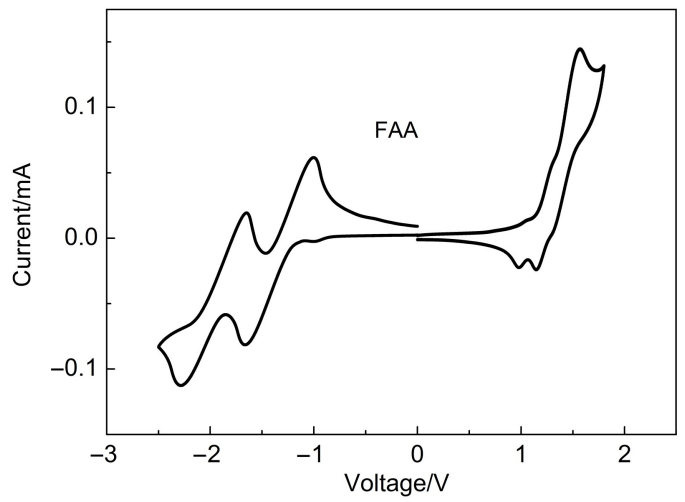

图6 FAA 的循环伏安图

Fig.6 Cyclic voltammograms of FAA

$\left(E_{\mathrm{ox}}\right)$ 和还原电势 $\left(E_{\mathrm{red}}\right)$ 分别为 1.13 和 $-1.21 \mathrm{~V}$, 由此 得出 FAA 的 HOMO 的能级为 $-5.85 \mathrm{eV}, \mathrm{LUMO}$ 能 级为 $-3.51 \mathrm{eV}$ 。

\section{3 热稳定性质}

本文采用热重分析法(TGA)和差示扫描量热法 (DSC) 对FAA 的热稳定性质进行了测试。如图 7(a) 所示, 以 5\%质量衰减为基准, 可以得出 FAA 的热 分解温度 $\left(T_{\mathrm{d}}\right)$ 为 $327^{\circ} \mathrm{C}$; 图 7(b) 为 $\mathrm{FAA}$ 的差示扫描 量热曲线, 从曲线中可知, 化合物没有显示出明 显的玻璃化转变温度 $\left(T_{\mathrm{g}}\right)$ 。该化合物良好的热稳定 性为其成膜性以及器件稳定性奠定了基础。

\section{4 载流子传输性质}

为了探讨 FAA 的载流子传输性能, 本文分别 设计了其空穴和电子的单载流子器件, 并测试得 到了两种器件的电流密度-电压特性曲线(图 8)。 其中, 单空穴型和单电子型器件的结构分别是 ITO/PEDOT:PSS (40 nm)/FAA (60 nm)/NPB (30 $\mathrm{nm}) / \mathrm{Al}(100 \mathrm{~nm})$ 和 ITO/ZnO $(30 \mathrm{~nm}) / \mathrm{FAA}(60 \mathrm{~nm}) /$ $\mathrm{TPBi}(30 \mathrm{~nm}) / \mathrm{LiF}(0.8 \mathrm{~nm}) / \mathrm{Al}(100 \mathrm{~nm})$ 。从图 8 中 可以看出, 两种单载流子器件的电流密度一电压曲 线非常接近, 没有很大差别, 说明 FAA 中空穴和 电子的载流子的注入与传输能力相当, 具有较好 的双极性载流子平衡传输特性, 同时也验证了葱 醌基团的引入可以有效改善芴类化合物的电子注 入与传输性能, 进而实现其载流子的平衡。

\section{5 电致发光性质}

本文采用溶液和真空蒸镀相结合的方法，制 备了将 $\mathrm{FAA}$ 掺杂于 $\mathrm{mCP}$ 主体材料中作为有机发光 层的一种有机电致发光器件, 其结构为 ITO/ PEDOT:PSS $(40 \mathrm{~nm}) / \mathrm{mCP}: \mathrm{FAA}(8 \%(w)) / \mathrm{TmPyPb}$ $(60 \mathrm{~nm}) / \mathrm{LiF}(0.8 \mathrm{~nm}) / \mathrm{Al}(100 \mathrm{~nm})$ 。图 9 为不同电压 下该OLED 器件的归一化电致发光光谱图。如图所 

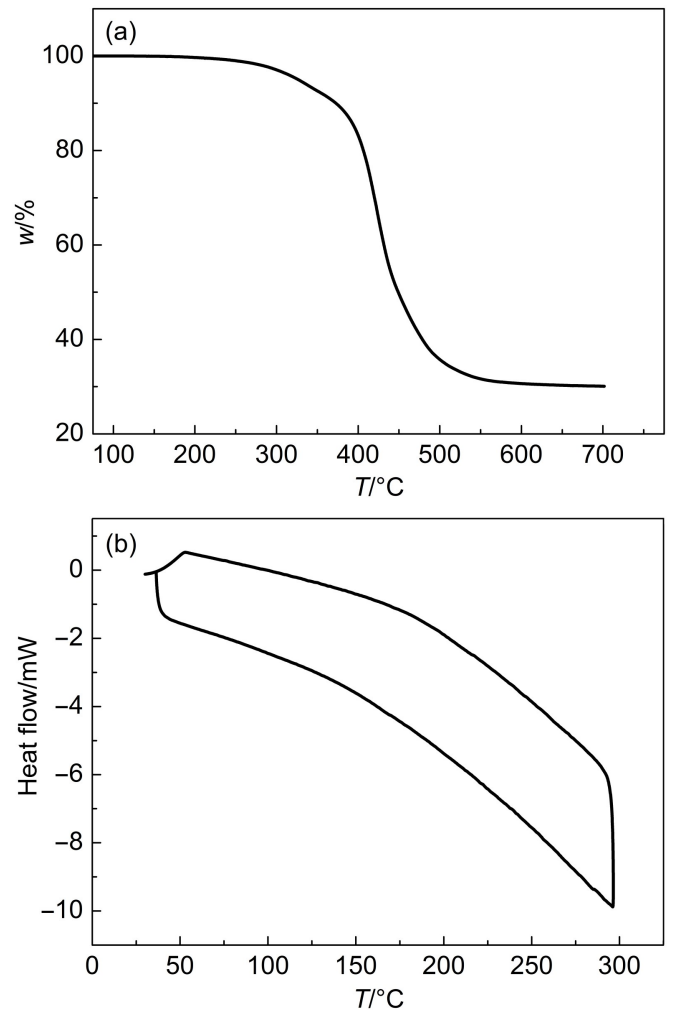

图 7 FAA 的(a)热重分析(TGA)曲线及 (b) 差示扫描量热法(DSC)曲线

Fig.7 (a) Thermogravimetric analysis (TGA) and (b) differential scanning calorimetry (DSC) curves of FAA

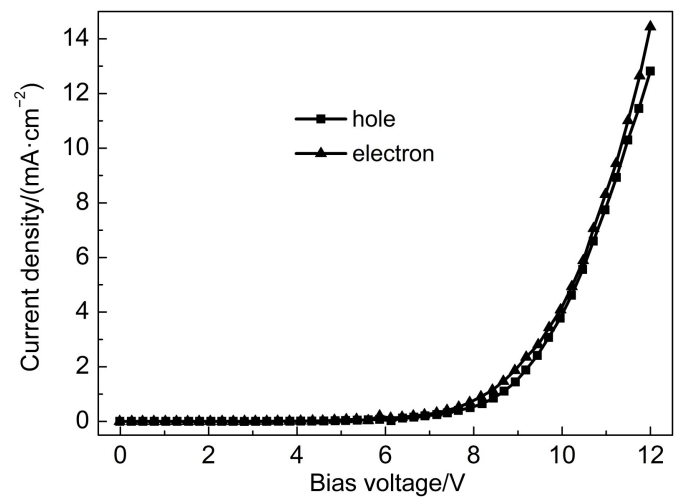

图 8 FAA 单空穴和单电子器件的电流密度一电压曲线图 Fig.8 Current density-voltage curves of hole only and electron only devices for FAA

示, 该器件显示出较宽的黄光发射 $(450-750 \mathrm{~nm})$, 随着电压的不断增大, 电致发光(EL)发射峰的峰形 基本不变, 其峰位置也几乎不变, 只发生了非常 微小的红移(从 $547 \mathrm{~nm}$ 到 $553 \mathrm{~nm}$ 波长范围内), 而 器件在不同电压时的 CIE 色坐标变化也很小(见表 1)，体现出很好的色纯度稳定性。

为了进一步讨论 OLED 器件的发光机制, 图 10 给出了器件各功能层的能级结构图。从图中可

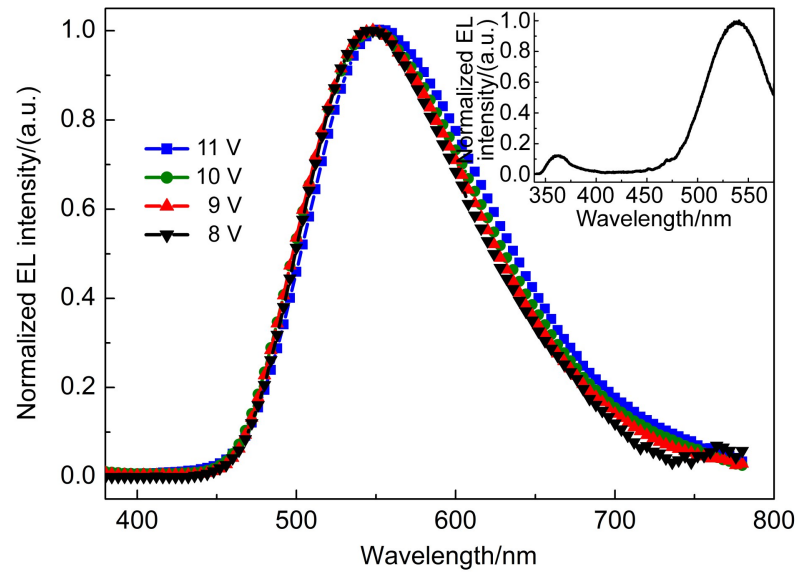

图9 不同电压下 OLED 器件的归一化电致发光光谱图

Fig.9 Normalized EL (electroluminescent) spectra of OLED device at different voltages

inset: normalized photoluminescent spectrum of FAA $(8 \%(w))$ doped into $\mathrm{mCP}$ film

表1 不同电压时 OLED 器件的 CIE 色坐标 Table 1 CIE coordination of the OLED at different voltages

\begin{tabular}{cc}
\hline Voltage/V & CIE coordination $(x, y)$ \\
\hline 8 & $0.4040,0.5345$ \\
9 & $0.4052,0.5305$ \\
10 & $0.4077,0.5252$ \\
11 & $0.4194,0.5202$ \\
\hline
\end{tabular}

CIE: Commission Internationale de L'Eclairage

知, $\mathrm{mCP}$ 和 FAA 的 $\mathrm{HOMO}$ 能级差值仅为 $0.05 \mathrm{eV}$, FAA 空穴陷阱效应可以忽略。但对于 LUMO 能级 来说, $\mathrm{mCP}$ 的 LUMO 能级比 FAA 高近 $0.9 \mathrm{eV}$, 所 以 FAA 可以在有机发光层中形成电子陷阱。另 外, 从图 9 的插图中, 我们可以看到 $\mathrm{mCP}: \mathrm{FAA}$ $(8 \%(w))$ 薄膜在 $360 \mathrm{~nm}$ 附近存在一个很小的主体材 料 $\mathrm{mCP}$ 的发射峰, 但在其 $\mathrm{EL}$ 光谱中并没有出现这 个峰, 说明器件中除了主客体材料间的能量传递

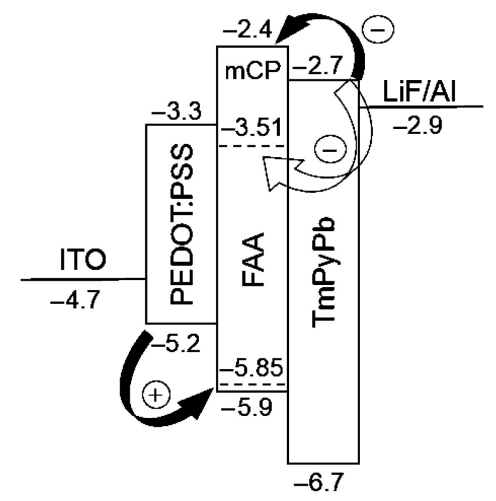

图 10 OLED 器件的能级 $(\mathrm{eV})$ 结构图

Fig.10 Energy level (eV) diagram of OLED device 
外, 还存在FAA 载流子陷阱俘获发光机制。

图 11 描述了 OLED 器件的电流密度一电压一亮 度特性曲线。由图可知, 在电压较低时, 器件的 电流密度随电压的增大以近似于线性的方式缓慢 增大; 当电压超过 $10 \mathrm{~V}$ 后, 电流密度有急剧的跃 升, 呈现出典型的二极管整流特性。该器件的启 亮电压约为 $7.4 \mathrm{~V}$, 在驱动电压为 $13 \mathrm{~V}$ 时, 达到最 大亮度 $1719 \mathrm{~cd} \cdot \mathrm{m}^{-2}$, 此时电流密度为 $354.19 \mathrm{~mA}$. $\mathrm{cm}^{-2}$ 。图 12 给出了该器件的电流效率-电流密度功率效率特性曲线, 在低电流密度时, 器件的电 流效率和功率效率都随电流密度增大而急剧上 升, 当电流密度为 $6.18 \mathrm{~mA} \cdot \mathrm{cm}^{-2}$ 时, 电流效率和 功率效率分别达到最大值 $1.66 \mathrm{~cd} \cdot \mathrm{A}^{-1}$ 和 $0.56 \mathrm{~lm}$. $\mathrm{W}^{-1}$; 随后, 电流效率和功率效率都会随着电流密 度的继续增大而下降。其中，较高的启亮电压以 及低器件效率, 主要是因为空穴由 PEDOT:PSS 层 注入到有机发光层时有较大的注入势垒 $(0.7 \mathrm{eV})$ 所 导致的。有望通过对该器件结构的不断优化, 并

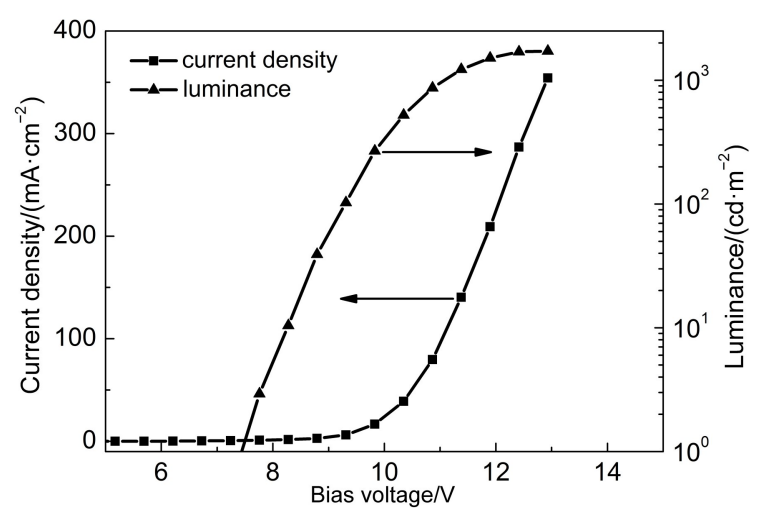

图 11 OLED 器件的电流密度-电压一亮度曲线图 Fig.11 Current density-voltage-luminance curves of OLED device

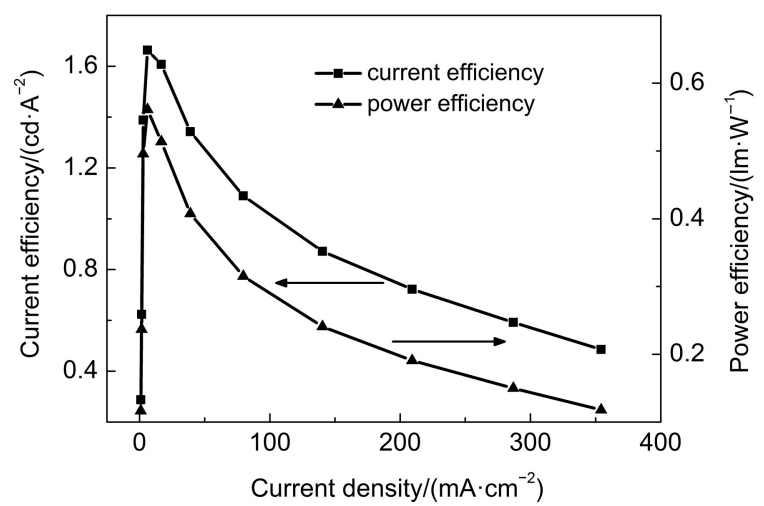

图 12 OLED 器件的电流效率-电流密度-功率效率曲线图

Fig.12 Current efficiency-current density-power efficiency curves of OLED device
选择更适合的主体材料, 进一步降低启亮电压, 提高器件效率。

\section{4 结 论}

本文在芴的 2 位碳上引入葱醌基团, 合成出一 种可溶液加工的葱醌/芴类双极性苂光材料 2-葱醌 基-9,9'-二异辛基荡。通过研究 FAA 载流子的传输 性能, 我们证明了其具有较好的双极载流子传输 特性, 从而说明了葱醌基团的引入能够实现芴类 化合物中载流子的平衡。本工作初步探索了基于 葱醌基团的芴类双极性苂光材料的光电性能, 后 续将通过进一步的结构设计, 引入延迟苂光性 能, 以期得到双极性、高效率的可溶液加工的有 机小分子 OLED 苂光材料。

\section{References}

(1) Baldo, M. A.; O'Brien, D. F.; You, Y.; Shoustikov, A.; Sibley, S.; Thompson, M. E.; Forrest, S. R. Nature 1998, 395 (6698), 151. doi: 10.1038/25954

(2) Muller, C. D.; Falcou, A.; Reckefuss, N.; Rojahn, M.; Wiederhirn, V.; Rudati, P.; Frohne, H.; Nuyken, O.; Becker, H.; Meerholz, K. Nature 2003, 421 (6925), 829. doi: 10.1038/ nature 01390

(3) Sun, Y. R.; Giebink, N. C.; Kanno, H.; Ma, B. W.; Thompson, M. E.; Forrest, S. R. Nature 2006, 440 (7086), 908. doi: 10.1038/ nature 04645

(4) Uoyama, H.; Goushi, K.; Shizu, K.; Nomura, H.; Adachi, C. Nature 2012, 492 (7428), 234. doi: 10.1038/nature11687

(5) Kaji, H.; Suzuki, H.; Fukushima, T.; Shizu, K.; Suzuki, K.; Kubo, S.; Komino, T.; Oiwa, H.; Suzuki, F.; Wakamiya, A.; Murata, Y.; Adachi, C. Nat. Commun. 2015, 6, 8. doi: 10.1038/ ncomms 9476

(6) Cai, M.; Xiao, T.; Hellerich, E.; Chen, Y.; Shinar, R.; Shinar, J. Adv. Mater. 2011, 23 (31), 3590. doi: 10.1002/adma.201101154

(7) Aizawa, N.; Pu, Y. J.; Watanabe, M.; Chiba, T.; Ideta, K.; Toyota, N.; Igarashi, M.; Suzuri, Y.; Sasabe, H.; Kido, J. Nat. Commun. 2014, 5, 7. doi: 10.1038/ncomms6756

(8) Höfle, S.; Schienle, A.; Bernhard, C.; Bruns, M.; Lemmer, U.; Colsmann, A. Adv. Mater. 2014, 26 (30), 5155. doi: 10.1002/ adma.201400332

(9) Zhang, X. W.; Yang, T.; Wei, Q.; Chen, Y. H.; Guo, X.; Xie, L. H.; Lai, W. Y.; Fan, Q. L.; Qian, Y.; Huang, W. RSC Adv. 2015, 5 (114), 94077. doi: 10.1039/c5ra19231e

(10) Liu, H.; Bai, Q.; Yao, L.; Hu, D. H.; Tang, X. Y.; Shen, F. Z.; Zhang, H. H.; Gao, Y.; Lu, P.; Yang, B.; Ma, Y. G. Adv. Funct. Mater. 2014, 24 (37), 5881. doi: 10.1002/adfm.201401183

(11) Oh, H. Y.; Kulshreshtha, C.; Kwon, J. H.; Lee, S. Org. Electron. 2010, 11 (10), 1624. doi: 10.1016/j.orgel.2010.07.005 
(12) Perumal, A.; Faber, H.; Yaacobi-Gross, N.; Pattanasattayavong, P.; Burgess, C.; Jha, S.; McLachlan, M. A.; Stavrinou, P. N.; Anthopoulos, T. D.; Bradley, D. D. C. Adv. Mater. 2015, 27 (1), 93. doi: 10.1002/adma.201403914

(13) Yook, K. S.; Lee, J. Y. Adv. Mater. 2014, 26 (25), 4218. doi: 10.1002/adma.201306266

(14) Chiba, T.; Pu, Y. J.; Kido, J. Adv. Mater. 2015, 27 (32), 4681. doi: 10.1002/adma.201501866

(15) Huang, M. H.; Lin, W. C.; Fan, C. C.; Wang, Y. S.; Lin, H. W.; Liao, J. L.; Lin, C. H.; Chi, Y. Org. Electron. 2015, 20, 36. doi: 10.1016/j.orgel.2015.01.019

(16) Cho, Y. J.; Yook, K. S.; Lee, J. Y. Adv. Mater. 2014, 26 (38), 6642. doi: 10.1002/adma.201402188

(17) Huang, T. H.; Lin, J. T.; Chen, L. Y.; Lin, Y. T.; Wu, C. C. Adv. Mater. 2006, 18 (5), 602. doi: 10.1002/adma.200502078

(18) Lin, S. L.; Chan, L. H.; Lee, R. H.; Yen, M. Y.; Kuo, W. J.; Chen, C. T.; Jeng, R. J. Adv. Mater. 2008, 20 (20), 3947. doi: 10.1002/adma.200801023

(19) Chaskar, A.; Chen, H. F.; Wong, K. T. Adv. Mater. 2011, 23 (34), 3876. doi: 10.1002/adma.201101848

(20) Duan, L. A.; Qiao, J. A.; Sun, Y. D.; Qiu, Y. Adv. Mater. 2011, 23 (9), 1137. doi: 10.1002/adma.201003816

(21) Hudson, Z. M.; Wang, Z. B.; Helander, M. G.; Lu, Z. H.; Wang, S. N. Adv. Mater. 2012, 24 (21), 2922. doi: 10.1002/ adma.201200927

(22) Lee, C. W.; Lee, J. Y. Adv. Mater. 2013, 25 (4), 596. doi: 10.1002/adma.201203180

(23) Hu, J. Y.; Pu, Y. J.; Satoh, F.; Kawata, S.; Katagiri, H.; Sasabe, H.; Kido, J. Adv. Funct. Mater. 2014, 24 (14), 2064. doi: 10.1002/adfm.201302907

(24) Wang, F. F.; Tao, Y. T.; Huang, W. Acta Chim. Sin. 2015, 73 (1), 9. [王芳芳, 陶友田, 黄 维. 化学学报, 2015, 73 (1), 9.] doi: $10.6023 / \mathrm{a} 14100716$

(25) Zhong, B. F.; Wang, S. R.; Xiao, Y.; Li, X. G. Prog. Chem. 2015, 27 (8), 986. [钟渤凡, 王世荣, 肖 殷, 李祥高. 化学进 展, 2015, 27 (8), 986.] doi: 10.7536/pc150151

(26) Ge, Z.; Hayakawa, T.; Ando, S.; Ueda, M.; Akiike, T.; Miyamoto, H.; Kajita, T.; Kakimoto, M. A. Chem. Mater. 2008, 20 (7), 2532. doi: 10.1021/cm7035458

(27) Gong, S. L.; Fu, Q.; Wang, Q.; Yang, C. L.; Zhong, C.; Qin, J. G.; Ma, D. G. Adv. Mater. 2011, 23 (42), 4956. doi: 10.1002/ adma.201102758

(28) Kong, S.; Xiao, L. X.; Liu, Y. L.; Chen, Z. J.; Qu, B.; Gong, Q. H. New J. Chem. 2010, 34 (9), 1994. doi: 10.1039/c0nj00011f

(29) Zhang, M.; Xue, S. F.; Dong, W. Y.; Wang, Q.; Fei, T.; Gu, C.; Ma, Y. G. Chem. Commun. 2010, 46 (22), 3923. doi: 10.1039/ c001170c

(30) Jiang, W.; Ban, X. X.; Sun, Y. M. RSC Adv. 2015, 5 (82), 66994. doi: 10.1039/c5ra12377a

(31) Jou, J. H.; Kumar, S.; Fang, P. H.; Venkateswararao, A.; Thomas, K. R. J.; Shyue, J. J.; Wang, Y. C.; Li, T. H.; Yu, H. H.
J. Mater. Chem. C 2015, 3 (10), 2182. doi: 10.1039/c4tc02547d

(32) Ye, J.; Chen, Z.; Fung, M. K.; Zheng, C. J.; Ou, X. M.; Zhang, X. H.; Yuan, Y.; Lee, C. S. Chem. Mater. 2013, 25 (13), 2630. doi: 10.1021/cm400945h

(33) Chen, C. T.; Wei, Y.; Lin, J. S.; Moturu, M. V. R. K.; Chao, W S.; Tao, Y. T.; Chien, C. H. J. Am. Chem. Soc. 2006, 128 (34), 10992. doi: 10.1021/ja062660v

(34) Chi, C. C.; Chiang, C. L.; Liu, S. W.; Yueh, H.; Chen, C. T.; Chen, C. T. J. Mater. Chem. 2009, 19 (31), 5561. doi: 10.1039/ b902910a

(35) Zhao, Y. L.; Duan, L.; Qiao, J.; Zhang, D. Q.; Wang, L. D.; Qiu, Y. Acta Phys. -Chim. Sin. 2010, 26 (3), 531. [赵云龙, 段 炼, 乔 娟, 张德强, 王立铎, 邱 勇. 物理化学学报, 2010, 26 (3), 531.] doi: 10.3866/PKU.WHXB20100328

(36) Tang, X. Y.; Bai, Q.; Peng, Q. M.; Gao, Y.; Li, J. Y.; Liu, Y. L.; Yao, L.; Lu, P.; Yang, B.; Ma, Y. G. Chem. Mater. 2015, 27 (20), 7050. doi: 10.1021/acs.chemmater.5b02685

(37) Mallesham, G.; Swetha, C.; Niveditha, S.; Mohanty, M. E.; Babu, N. J.; Kumar, A.; Bhanuprakash, K.; Rao, V. J. J. Mater. Chem. C. 2015, 3 (6), 1208. doi: 10.1039/c4tc01753f

(38) Li, J. W.; Qian, Y.; Xie, L. H.; Yi, Y. P.; Li, W. W.; Huang, W. J. Phys. Chem. C 2015, 119 (4), 2133. doi: 10.1021/jp5089433

(39) Martin, R. E.; Diederich, F. Angew. Chem. Int. Ed. 1999, 38 (10), 1350. doi: 10.1002/(sici)1521-3773(19990517)38:10< 1350::aid-anie1350>3.0.co;2-6

(40) Lee, S. H.; Nakamura, T.; Tsutsui, T. Org. Lett. 2001, 3 (13), 2005. doi: $10.1021 / \mathrm{ol} 1010069 \mathrm{r}$

(41) Setayesh, S.; Grimsdale, A. C.; Weil, T.; Enkelmann, V.; Müllen, K.; Meghdadi, F.; List, E. J. W.; Leising, G. J. Am. Chem. Soc. 2001, 123 (5), 946. doi: 10.1021/ja0031220

(42) Xiao, L. X.; Hu, S. Y.; Kong, S.; Chen, Z. J.; Qu, B.; Gong, Q. H. Acta Phys. -Chim. Sin. 2011, 27 (4), 977. [肖立新, 胡双元, 孔 胜, 陈志坚, 曲 波, 龚旗煌. 物理化学学报, 2011, 27 (4), 977.] doi: 10.3866/PKU.WHXB20110325

(43) Huo, Y. P.; Fang, X. M.; Huang, B. H.; Zhang, K.; Nie, X. L.; Zeng, H. P. Chin. J. Org. Chem. 2012, 32 (7), 1169. [霍延平, 方 小明, 黄宝华, 张 焜, 聂晓李, 曾和平. 有机化学, 2012, 32 (7), 1169.] doi: 10.6023/cjoc201204021

(44) Chu, Z. Z.; Wang, D.; Zhang, C.; Zou, D. C. Acta Phys. -Chim. Sin. 2012, 28 (8), 2000. [初增泽, 王 丹, 张 超, 邹德春. 物理 化学学报, 2012, 28 (8), 2000.] doi: 10.3866/PKU. WHXB201206071

(45) Kamtekar, K. T.; Wang, C. S.; Bettington, S.; Batsanov, A. S.; Perepichka, I. F.; Bryce, M. R.; Ahn, J. H.; Rabinal, M.; Petty, M. C. J. Mater. Chem. 2006, 16 (39), 3823. doi: 10.1039/ b604543j

(46) Lai, M. Y.; Chen, C. H.; Huang, W. S.; Lin, J. T.; Ke, T. H.; Chen, L. Y.; Tsai, M. H.; Wu, C. C. Angew. Chem. Int. Ed. 2008, 47 (3), 581. doi: 10.1002/anie.200704113

(47) Li, Y.; Li, A. Y.; Li, B. X.; Huang, J.; Zhao, L.; Wang, B. Z.; Li, J. W.; Zhu, X. H.; Peng, J. B.; Cao, Y.; Ma, D. G.; Roncali, J. 
Org. Lett. 2009, 11 (22), 5318. doi: 10.1021/o19022563

(48) Yu, D. H.; Zhao, F. C.; Zhang, Z.; Han, C. M.; Xu, H.; Li, J.; Ma, D. G.; Yan, P. F. Chem. Commun. 2012, 48 (49), 6157. doi: $10.1039 / \mathrm{c} 2 \mathrm{cc} 31066 \mathrm{j}$

(49) Feng, X. J.; Chen, S. F.; Ni, Y.; Wong, M. S.; Lam, M. M. K.; Cheah, K. W.; Lai, G. Q. Org. Electron. 2014, 15 (1), 57. doi: 10.1016/j.orgel.2013.10.019

(50) Zhang, Q. S.; Kuwabara, H.; Potscavage, W. J.; Huang, S. P.; Hatae, Y.; Shibata, T.; Adachi, C. J. Am. Chem. Soc. 2014, 136
(52), 18070. doi: 10.1021/ja510144h

(51) Sonntag, M.; Kreger, K.; Hanft, D.; Strohriegl, P.; Setayesh, S.; de Leeuw, D. Chem. Mater. 2005, 17 (11), 3031. doi: 10.1021/ $\mathrm{cm} 047750 \mathrm{i}$

(52) Cho, S. Y.; Grimsdale, A. C.; Jones, D. J.; Watkins, S. E.; Holmes, A. B. J. Am. Chem. Soc. 2007, 129 (39), 11910. doi: $10.1021 / \mathrm{ja} 074634 \mathrm{i}$

(53) Frisch, M.; Trucks, G.; Schlegel, H.; et al. Gaussian 09, Revision A.1; Gaussian Inc.: Wallingford, CT, 2009. 